\title{
ACTH-secreting medullary thyroid cancer: a case series
}

\author{
Ayanthi A Wijewardene', Sarah J Glastras,3,4, Diana L Learoyd ${ }^{2,4}$, Bruce G Robinson ${ }^{2,4}$ \\ and Venessa H M Tsang2,4
}

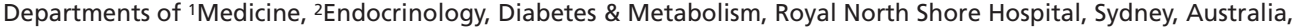
${ }^{3}$ Kolling Institute of Medical Research, and ${ }^{4}$ Sydney Medical School, University of Sydney, Sydney, Australia
Correspondence should be addressed to A A Wijewardene Email

ayanthi.w@gmail.com

\section{Summary}

Medullary thyroid cancer (MTC) is a rare neuroendocrine tumour that originates from the parafollicular cells of the thyroid gland. The most common presentation of MTC is with a single nodule; however, by the time of diagnosis, most have spread to the surrounding cervical lymph nodes. Cushing's syndrome is a rare complication of MTC and is due to ectopic adrenocorticotrophic hormone (ACTH) secretion by tumour cells. Cushing's syndrome presents a challenging diagnostic and management issue in patients with MTC. Tyrosine kinase inhibitors (TKI) previously used for the management of metastatic MTC have become an important therapeutic option for the management of ectopic ACTH in metastatic MTC. The article describes three cases of ectopic ACTH secretion in MTC and addresses the significant diagnostic and management challenges related to Cushing's syndrome in metastatic MTC.

\section{Learning points:}

- Medullary thyroid cancer (MTC) is a rare neuroendocrine tumour.

- Cushing's syndrome is a rare complication of MTC that has a significant impact on patients' morbidity and mortality.

- Tyrosine kinase inhibitors (TKI) provide an important therapeutic option for the management of ectopic ACTH in metastatic MTC.

\section{Background}

Three cases of medullary thyroid cancer (MTC) with ectopic ACTH secretion have been described. Ectopic ACTH secretion is a rare presentation of MTC and can be clinically suspected by the development of Cushing's syndrome. Only approximately 50 cases have been published on Cushing syndrome in MTC. Presentation of ectopic ACTH secretion in this context presents significant management challenges related to both the control of metastatic MTC and the Cushing's syndrome, with its associated extreme morbidity.

\section{Case presentation}

\section{Case 1}

A 35-year-old male presented in 2014 with incidental supraclavicular lymphadenopathy on a computer tomography (CT) chest after investigation of anterior chest wall pain and rib fracture, on a background of type 1 diabetes. He was subsequently diagnosed with metastatic MTC. Six months after a total thyroidectomy, he became increasingly Cushingnoid with weight gain and a plethoric appearance. 


\section{Case 2}

A 53-year-old male presented in 2002 with increasing back pain associated with Cushingoid features of weight gain, facial plethora, abdominal striae and proximal myopathy.

\section{Case 3}

A 38-year-old male was investigated in 2003 for a palpable mass in his left lower neck. He was diagnosed with metastatic medullary thyroid cancer. During the work-up of his treatment, he became increasingly Cushingoid with worsening hypertension, abdominal distension and striae.

\section{Investigation}

\section{Case 1}

Fine needle aspiration (FNA) of the supraclavicular lymph node revealed MTC consistent with his elevated plasma calcitonin of $1002 \mathrm{ng} / \mathrm{L}$ (normal range (NR) <20). Gallium-68 (Ga-68) DOTATATE positron emission tomography (PET) scan was then performed, which showed supraclavicular, upper mediastinal and paraaortic lymphadenopathy with DOTATATE avidity.

A total thyroidectomy and bilateral central neck dissection were performed, with histology confirming multifocal bilateral MTC with immunohistochemistrypositive staining for calcitonin. Tumours ranged from $0.5 \mathrm{~mm}$ to $22 \mathrm{~mm}$ in diameter and involved 16 of $18 \mathrm{lymph}$ nodes. Due to the normal serum calcium $(2.43 \mathrm{mmol} / \mathrm{L})$, parathyroid hormone $(7.9 \mathrm{ng} / \mathrm{L})$ and plasma metanephrine (0.31 nmol/L) levels, multiple endocrine neoplasia (MEN) syndrome, a genetic syndrome that is found in $25 \%$ of patients with MTC, was considered very unlikely. Germline testing for the Rearranged in Transfection (RET) gene revealed no mutation.

Surveillance for tumour recurrence was assessed by interval CT scans and plasma calcitonin, which initially improved to $400 \mathrm{ng} / \mathrm{L}(\mathrm{NR}<20)$ after surgery. Six months later, he developed increasing lethargy and weight gain with a plethoric appearance. Blood pressure was mildly elevated at $148 / 88 \mathrm{mmHg}$ and glycaemic control deteriorated (HbA1c: 7.9\%). 24-h urinary free cortisol was elevated at $562 \mathrm{nmol}(\mathrm{NR}<300)$, and ACTH was inappropriately detectable at $8.8 \mathrm{pmol} / \mathrm{L}$ (NR: 0-12). A high-dose dexamethasone suppression test failed to suppress with a serum cortisol level of $339 \mathrm{nmol} / \mathrm{L}$, consistent with ectopic ACTH-dependent Cushing's syndrome. ACTH staining of the original thyroid tissue was negative.

\section{Case 2}

CT of the lumbar spine revealed destructive lesions in L3 and the left pedicle and body of L4. Laminectomy was performed and the presence of small cell neuroendocrine tumour with positive chromogranin staining was found. His pre-operative calcitonin level was elevated at $2200 \mathrm{ng} / \mathrm{L}(\mathrm{NR}<20.0)$. A total thyroidectomy and paratracheal lymph node clearance were identified, a $9 \mathrm{~mm}$ MTC in the left lobe of the thyroid and lymph node involvement. Immunohistochemistry staining of thyroid tissue was positive for calcitonin, chromogranin and carcinoembryonic antigen (CEA). ACTH staining of the thyroid gland was negative as seen in Fig. 1. CT scan of the chest, abdomen and pelvis showed only the localised bony lesion. Cushing's syndrome was confirmed with an
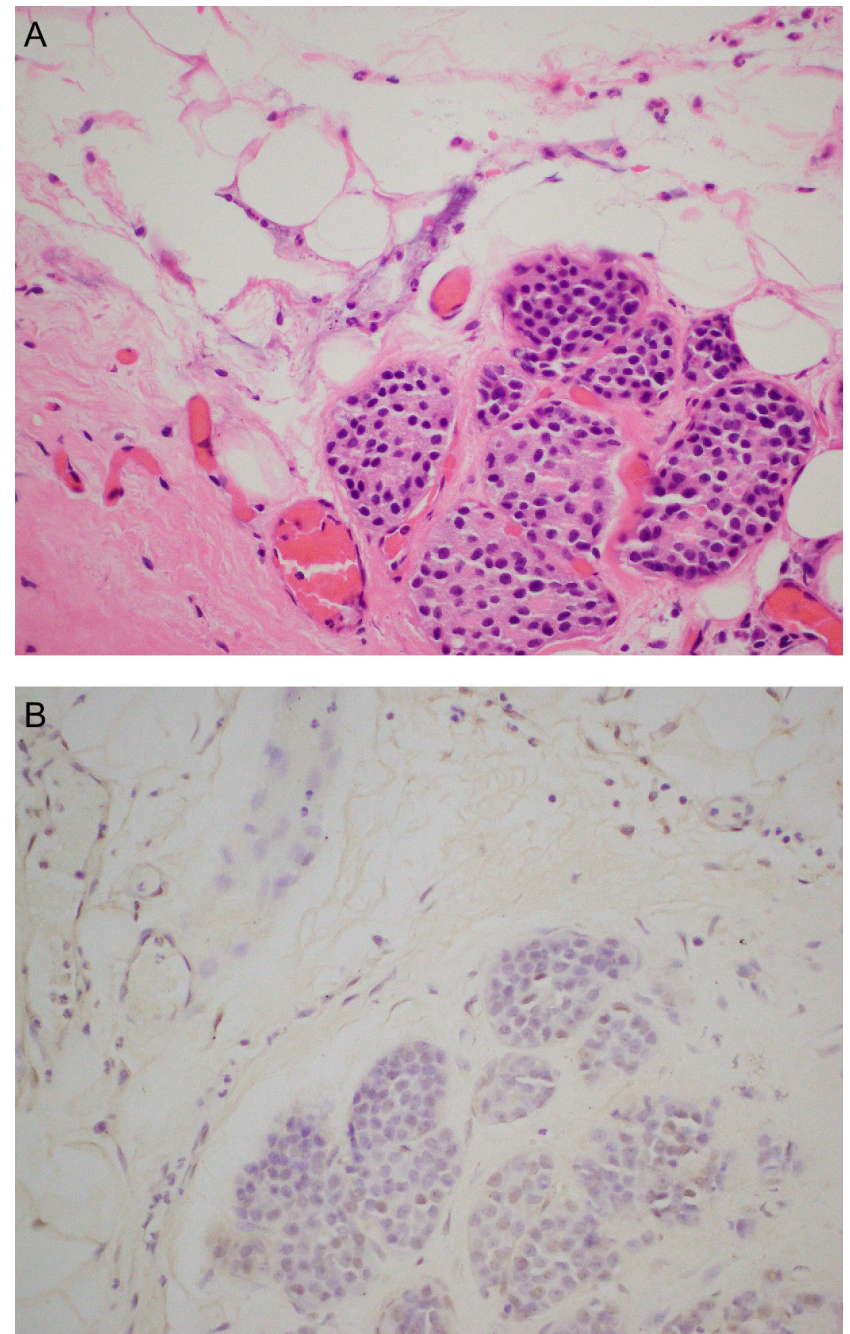

Figure 1

(A) Haematoxylin and eosin staining of MTC and (B) negative ACTH staining of MTC. 
elevated 24-h urinary cortisol at $15708 \mathrm{nmol}(\mathrm{NR}<300$ ), early morning cortisol of $2045 \mathrm{nmol}$ (NR: 25-695) and an inappropriately normal ACTH level of $8.7 \mathrm{pmol} / \mathrm{L}$ (NR: $0-12$ ).

\section{Case 3}

The initial FNA of the neck nodule was normal; however, subsequent excision biopsy was consistent with MTC. The tumour cells stained positively for calcitonin and chromogranin. Disease staging was performed with CT scan of the neck and chest, which showed a substernal goitre with a $7 \mathrm{~cm}$ left-sided nodule and multiple small lesions throughout the lungs consistent with Stage IV disease. Calcitonin was elevated to $5400 \mathrm{nmol} / \mathrm{L}$ (NR $<720)$. A total thyroidectomy with left neck node excision was performed. Germline genetic testing was negative for RET mutation.

An interval CT scan 2 years after thyroidectomy showed spread of disease with a new $42 \mathrm{~mm}$ liver lesion, new pulmonary nodules and further cervical and mediastinal lymphadenopathy. No abnormalities in the adrenal glands were detected on imaging.

\section{Treatment}

\section{Case 1}

Ketoconazole was initiated at $200 \mathrm{mg}$ twice-daily and increased to $400 \mathrm{mg}$ twice-daily as he remained floridly Cushingoid with a weight gain of $30 \mathrm{~kg}$, plethoric appearance and abdominal striae. His condition was complicated by recurrent pneumonia. Calcitonin, which had previously been stable, increased from $477 \mathrm{ng} / \mathrm{L}$ to $1194 \mathrm{ng} / \mathrm{L}(\mathrm{NR}<20)$, indicating further progression of the disease. A tyrosine kinase inhibitor (TKI), vandetanib, was commenced to manage the progressive MTC. Vandetanib is a multi-kinase inhibitor, with effects against RET kinase, vascular endothelial growth factor receptor (VEGFR) types 2 and 3 and epidermal growth factor receptor (EGFR). On vandetanib, his electrolytes including potassium were monitored three times a week and remained between 4.0 and $5.0 \mathrm{mmol} / \mathrm{L}$. Though dosage of $300 \mathrm{mg}$ daily was commenced initially, the dose was subsequently reduced to $200 \mathrm{mg}$ due to prolonged QTc demonstrated on ECG. An acneiform rash (grade 1) also developed. While on combination of vandetanib and ketoconazole, there was notable clinical improvement with reduced lethargy, there was no suggestion of progressive disease either radiologically or biochemically and plasma calcitonin reduced to $570 \mathrm{ng} / \mathrm{L}(\mathrm{NR}<20)$. Thryoxine dose required to be increased from $150 \mu$ g daily to $225 \mu$ g daily on vandetanib.

Due to side effect of prolonged QTc with vandetanib and ongoing need to manage the Cushing's syndrome, a bilateral adrenalectomy was attempted. However, significant hypoxia during attempts at intubation made it unsafe to proceed with adrenalectomy, and so, the operation was abandoned. In a further attempt to control the Cushing's syndrome, ketoconazole was replaced with mitotane. Mitotane was titrated from a starting dose of $500 \mathrm{mg}$ twice daily and increased to $1000 \mathrm{mg}$ twice daily. He had a significant response with weight loss, improved glycaemic control (HbA1c: 6.7\%) and his 24-h urine cortisol reduced to the normal range ( $98 \mathrm{nmol}$ in $24 \mathrm{~h}$ ).

A follow-up PET scan 12 months after thyroidectomy showed progression of disease with widespread DOTATATE-avid lymphadenopathy in the neck, thorax and abdomen with elevation of calcitonin to $1171 \mathrm{ng} / \mathrm{L}$ (NR <20). Vandetanib is metabolised by the CYP3A4 pathway in which mitotane is an enzyme inducer; this may have impacted vandetanib drug levels and hence efficacy. Vandetanib was ceased for 6 weeks due to disease progression and an alternative TKI, cabozantinib, was considered. Cabozantinib has effects against c-Met and VEGFR type 2, with Phase III trials showing efficacy against progressive unresectable or metastatic MTC. Development of haemoptysis and new lung metastasis ruled out participation in available cabozantinib clinical trials. Vandetanib was recommenced due to worsening of Cushing's syndrome off treatment with increasing lethargy and plethoric facies.

\section{Case 2}

Cushing's syndrome due to ectopic ACTH secretion was initially managed with local irradiation to the lumbar spine with 40 Gy over 4 weeks to the lumbar spine, L2-L4. Ketoconazole was then commenced, resulting in the normalisation of urinary free cortisol to $137 \mathrm{nmol} / 24 \mathrm{~h}(\mathrm{NR}<300)$. The symptoms due to myopathy dramatically improved.

An asymptomatic palpable lymph node was detected in the left posterior triangle one year after the laminectomy. A biopsy of the node was consistent with MTC requiring excision of the lymph node. One month later, there was evidence of recurrent Cushing's syndrome with raised 24-h urinary free cortisol $(8977 \mathrm{nmol}$, NR $<300)$ and ACTH $(148 \mathrm{ng} / \mathrm{L}, \mathrm{NR}<46)$. 
Ketoconazole was recommenced and titrated to $200 \mathrm{mg}$ twice daily, though there was poor drug compliance due to gastrointestinal side effects. Tyrosine kinase inhibitors were not available for MTC management at the time.

\section{Case 3}

The patient was commenced on a Phase III double-blind, randomised, placebo-controlled vandetanib trial. During the course of treatment, the patient developed worsening hypertension, abdominal distension and striae. He also reported persistent back pain. Investigations for secondary causes of hypertension revealed an elevated 24-h urinary free cortisol at $2230 \mathrm{nmol} /$ day $(\mathrm{NR}<300)$ and an elevated ACTH of $170 \mathrm{ng} / \mathrm{L}(\mathrm{NR}<46)$. Cushing's syndrome was confirmed with a non-suppressed morning cortisol of $780 \mathrm{nmol} / \mathrm{L}$ after a four-day low-dose dexamethasone suppression test and a persistently elevated 24-h urinary free cortisol of $1860 \mathrm{nmol} /$ day (NR <300). X-rays of his spine showed multiple vertebral fractures. Ketoconazole was commenced at $200 \mathrm{mg}$ twice daily and titrated up to $200 \mathrm{mg}$ three times a day. He was commenced on perindopril, telmisartan, indapamide and metoprolol to control his blood pressure. His clinical condition warranted unblinding from the study drug and placing him on the active drug. He was commenced on vandetanib $300 \mathrm{mg} /$ day. A 24-h urinary free cortisol improved to $260 \mathrm{nmol} /$ day. Ketoconazole was able to reduce to $200 \mathrm{mg} \mathrm{BD}$, and he required only metoprolol alone for blood pressure control. His thyroxine dose increased from $100 \mu \mathrm{g}$ to $200 \mu \mathrm{g}$ daily.

Bloods were monitored regularly while on vandetanib, and his potassium was replaced orally to remain within normal levels. Renal impairment was noted with increase in creatinine from $107 \mu \mathrm{mol} / \mathrm{L}$ to $352 \mu \mathrm{mol} / \mathrm{L}$. Vandetanib was withheld for 2 weeks and the creatinine improved to $122 \mu \mathrm{mol} / \mathrm{L}$. Vandetanib was recommenced at $200 \mathrm{mg}$ daily, the $\mathrm{Cr}$ remained initially stable but subsequently increased, and the vandetanib was reduced to $100 \mathrm{mg}$ daily. Ketoconazole was attempted to be reduced to $200 \mathrm{mg}$ daily; however, due to patient feeling symptomatically worse with bloating, he remained on $200 \mathrm{mg}$ twice daily.

\section{Outcome and follow-up}

\section{Case 1}

Cushing's manifestations improved on the combination of vandetanib and mitotane with urinary free cortisol remaining within normal limits $(243 \mathrm{nmol} / 24 \mathrm{~h}$, NR $<300$ ). Six months later, the patient was admitted with severe community-acquired pneumonia. Despite noninvasive ventilation and intravenous antibiotics, he died of sepsis.

\section{Case 2}

Further disease progression was seen with new mediastinal lymphadenopathy on CT scan, and the calcitonin level rose to $4140 \mathrm{ng} / \mathrm{L}(\mathrm{NR}<20)$. Despite surgical removal of the metastatic mediastinal lymph nodes and continuation of ketoconazole, there was rapid progression of Cushing's syndrome. Severe bilateral proximal myopathy, steroidinduced diabetes and cellulitis supervened with subsequent abscess formation in his left arm. The patient was severely debilitated and required admission into a high-level care residential facility, where he eventually died.

\section{Case 3}

The disease remained stable for a further 18 months, with no progression of disease noted on 3 monthly CT scans. In 2010, disease progression was evident both clinically by worsened hypertension and the development of diabetes mellitus and biochemically with increasing calcitonin levels $(3853 \mathrm{nmol} / \mathrm{L}, \mathrm{NR}<20)$. The liver metastases increased in size as demonstrated on CT scan, and 24-h urinary free cortisol increased to $1600 \mathrm{nmol}$ (NR $<300$ ). Metyrapone $750 \mathrm{mg}$ daily was added to maximally titrated ketoconazole. Vandetanib was eventually ceased due to QTc prolongation to $549 \mathrm{~ms}$ on the lowest dose of $100 \mathrm{mg}$ daily. Bilateral adrenalectomy was considered, though perioperative morbidity was considered too great due to the burden of disease. His Cushing's remained slowly progressive, though the metyrapone was initially poorly tolerated with nausea, was eventually able to be increased to $1500 \mathrm{mg}$ a day in six doses. His electrolytes were monitored, and potassium was replaced orally. Ketoconazole was ceased due to deranged liver function tests (GGT: 194 and ALP: 184), and no evidence of obstruction on liver ultrasound. Mitotane was added at $250 \mathrm{mg}$ daily and titrated to $1 \mathrm{~g}$ BD. Despite ongoing therapy, the disease continued to progress with marked proximal myopathy and significantly impaired mobility. The patient died in early 2011 .

\section{Discussion}

MTC is a rare malignancy that accounts for approximately $5-10 \%$ of all thyroid cancers (1). The majority of MTCs are sporadic; however, $25 \%$ of the patients have familial 
endocrine syndromes associated with $R E T$ proto-oncogene mutation (2). MTC is usually diagnosed with fine-needle aspiration, and MTC tumour-specific biomarkers such as calcitonin and carcinoembryonic antigen (CEA) increase testing sensitivity (3). MTC is localised to the thyroid gland and surrounding cervical lymph nodes in $70 \%$ of patients; however, up to $10 \%$ have distant metastases at the time of presentation (1). The first-line treatment for MTC is surgical resection; however, patients with metastatic disease at diagnosis are rarely cured. The tenyear survival in MTC is significantly reduced in patients with metastatic disease as compared with overall survival in all cases of MTC (73\% vs 91\% respectively) (4).

Ectopic ACTH secretion explains up to $18 \%$ of Cushing's syndrome cases and is a result of ACTH or CRH secretion outside the pituitary gland or hypothalamus respectively. A review by Barbosa and coworkers found only $0.7 \%$ of patients with MTC develops ectopic Cushing's syndrome and MTC accounts for approximately $2.2-7.5 \%$ of patients with ectopic ACTH (5). Clinical features, as seen in the three cases described, are typical of excess cortisol including weight gain, abdominal striae, lethargy, hypertension, new-onset diabetes and proximal myopathy. The diagnosis is made by documenting biochemical evidence of cortisol excess, which is usually confirmed with the 24-h urinary free cortisol measurement and inappropriately elevated ACTH levels during dexamethasone suppression testing. Confirming that MTC is the source of ectopic ACTH secretion can be difficult as MTC cells do not always show ACTH staining by immunohistochemistry, which may be due to reduced storage of ACTH despite increased secretion (6). Recent development of Gallium-68 (Ga-68) DOTATATE PET scans has allowed for improved detection of neuroendocrine tumours including MTC, these scans, however, were not available in the earlier Cases 2 and 3. The role of bilateral inferior petrosal sinus sampling is considered unnecessarily invasive in the context of patients being clinically Cushingoid with elevated free urinary cortisol and known history of metastatic MTC.

Management of Cushing's syndrome in MTC is crucial as secondary complications of hypercortisolism account for $50 \%$ of the mortality in MTC with ectopic Cushing's syndrome (6). Management is often delayed due to difficulties in establishing a diagnosis. Historically, management was limited to debulking metastatic disease and anti-adrenal therapies including medical therapy with ketoconazole, mitotane and metyrapone. Surgical intervention with bilateral adrenalectomy can be offered to some patients, but is a complex procedure in the setting of metastatic MTC. More recently, systemic therapy with TKIs offers a further management strategy for disease control of metastatic, unresectable disease or progressive, metastatic disease (2).

Vandetanib is an oral TKI that has been successfully used in the management of metastatic MTC with improved progression-free survival as compared to placebo (hazard ratio 0.46). The largest Phase III trial showed that the median treatment duration until disease progression in the vandetanib-treated group was longer compared to placebo (90.1 weeks vs 39.9 weeks respectively) (7). In addition, vandetanib has been found to have the extra benefit of reducing ectopic Cushing's syndrome in patients with metastatic MTC as described in Cases 1 and 3. Vandetanib has been shown to control ACTH secretion in recent case reports in both adult and adolescent populations $(6,8)$. Vandetanib is thought to have inhibitory actions on MTC oncogenes including the RET receptor, vascular endothelial growth factor receptor 2 and epidermal growth factor receptor, which in turn inhibit cell proliferation pathways (6). Control of ACTH secretion despite ongoing tumour progression, may be due to vandetanib anti-secretory effects (8). As seen in Cases 1 and 3, the most significant limitation of vandetanib use (like other TKIs) is its adverse effects, which include electrolyte derangement, cardiotoxicity, myelosuppression, arterial thromboembolism and hepatotoxicity. High-grade QTc prolongation has been reported in $12 \%$ of patients using vandetanib for thyroid cancer (9). Ketoconazole and mitotane can both result in QTC prolongation requiring close monitoring of ECG when combined with vandetanib. Vandetanib is metabolised by the CYP3A4 pathway, which can cause drug interaction with other CYP3A4 inducers such as mitotane influencing the vandetanib drug levels and efficacy. Currently, selection of TKI, with recent availability of cabozantinib and lenvatinib, is primarily determined by the side effect profile and availability of clinical trials.

Cushing's syndrome secondary to ectopic ACTH secretion is a rare complication of metastatic MTC. It is important for clinicians to be aware that Cushing's syndrome can sometimes be the presenting feature of metastatic MTC (2). Despite potentially serious adverse effects, TKIs have become an important therapeutic option for the management of ectopic ACTH in metastatic MTC. Nonetheless, this presentation is associated with significant morbidity and remains life threatening. 
Endocrinology

Diabetes \& Metabolism

CASE REPORTS
A A Wijewardene

and others
ACTH secreting medullary

thyroid cancer
ID: 16-0110; May 2017

DOI: 10.1530/EDM-16-0110
Declaration of interest

The authors declare that there is no conflict of interest that could be perceived as prejudicing the impartiality of the research reported.

\section{Funding}

This research did not receive any specific grant from any funding agency in the public, commercial or not-for-profit sector.

\section{Patient consent}

Consent was obtained prior to treatment. All patients are deceased.

\section{Author contribution statement}

The first author primarily wrote the article. All physicians responsible for the patients care are authors in the article. All authors provided clinical guidance in the writing of the article and editing the article.

\section{References}

1 Mackenzie EJ \& Mortimer RH 2004 6: Thyroid nodules and thyroid cancer. Medical Journal of Australia 180 242-247.

2 Wells SA Jr, Asa SL, Dralle H, Elisei R, Evans DB, Gagel RF, Lee N, Machens A, Moley JF, Pacini F, et al. 2015 Revised american thyroid association guidelines for the management of medullary thyroid carcinoma. Thyroid 25 567-610. (doi:10.1089/thy.2014.0335)
3 Bhanot P, Yang J, Schnadig VJ \& Logroño R 2007 Role of FNA cytology and immunochemistry in the diagnosis and management of medullary thyroid carcinoma: report of six cases and review of the literature. Diagnostic Cytopathology 35 285-292. (doi:10.1002/ dc.20502)

4 Rendl G, Manzl M, Hitzl W, Sungler P \& Pirich C 2008 Long-term prognosis of medullary thyroid carcinoma. Clinical Endocrinology 69 497-505. (doi:10.1111/j.1365-2265.2008.03229.x)

5 Barbosa SL, Rodien P, Leboulleux S, Niccoli-Sire P, Kraimps JL, Caron P, Archambeaud-Mouveroux F, Conte-Devolx B, Rohmer V \& Groupe d'Etude des Tumeurs Endocrines 2005 Ectopic adrenocorticotropic hormone-syndrome in medullary carcinoma of the thyroid: a retrospective analysis and review of the literature. Thyroid $\mathbf{1 5}$ 618-623. (doi:10.1089/thy.2005.15.618)

6 Nella AA, Lodish MB, Fox E, Balis FM, Quezado MM, Whitcomb PO, Derdak J, Kebebew E, Widemann BC \& Stratakis CA 2014 Vandetanib successfully controls medullary thyroid cancer-related Cushing syndrome in an adolescent patient. Journal of Clinical Endocrinology and Metabolism 99 3055-3059. (doi:10.1210/jc.2013-4340)

7 Wells SA Jr, Robinson BG, Gagel RF, Dralle H, Fagin JA, Santoro M, Baudin E, Elisei R, Jarzab B, Vasselli JR, et al. 2012 Vandetanib in patients with locally advanced or metastatic medullary thyroid cancer: a randomized, double-blind phase III trial. Journal of Clinical Oncology 30 134-141. (doi:10.1200/JCO.2011.35.5040)

8 Baudry C, Paepegaey AC \& Groussin L 2013 Reversal of Cushing's syndrome by vandetanib in medullary thyroid carcinoma. New England Journal of Medicine 369 584-586. (doi:10.1056/NEJMc1301428)

9 Zang J, Wu S, Tang L, Xu X, Bai J, Ding C, Chang Y, Yue L, Kang E \& He J 2012 Incidence and risk of QTc interval prolongation among cancer patients treated with vandetanib: a systematic review and meta-analysis. PLoS ONE 7 e30353. (doi:10.1371/journal. pone.0030353

Received in final form 6 March 2017

Accepted 28 March 2017 